\title{
Weekly paclitaxel for heavily treated advanced or recurrent gastric cancer refractory to fluorouracil, irinotecan, and cisplatin
}

\author{
Rai Shimoyama ${ }^{1,2}$, Hirofumi Yasui ${ }^{1}$, Narikazu Boku ${ }^{1}$, Yusuke Onozawa ${ }^{1}$, Shuichi Hironaka ${ }^{1}$, \\ Akira Fukutomi ${ }^{1}$, Kentaro Yamazaki $^{1}$, Keisei Taku ${ }^{1}$, ${\text { Takashi } \text { Kojima }^{1} \text {, Nozomu Machida }}^{1}$, Akiko Todaka ${ }^{1}$, \\ Hideharu Tomita $^{1}$, Takeshi Sakamoto ${ }^{1}$, and Takahiro Tsushima ${ }^{1}$ \\ ${ }^{1}$ Division of Gastrointestinal Oncology, Shizuoka Cancer Center, 1007 Shimonagakubo, Nagaizumi-cho, Shizuoka 411-8777, Japan \\ ${ }^{2}$ Department of General Surgery, Shonankamakura General Hospital, Kanagawa, Japan
}

\begin{abstract}
Background. Although triweekly administration of paclitaxel is approved for gastric cancer in Japan, currently, the drug is often delivered with a weekly schedule because of the equivalent efficacy and lesser toxicity of this dosing schedule as compared with the triweekly administration schedule. Weekly administration of paclitaxel as second-line or first-line chemotherapy for gastric cancer has been reported to yield a response rate of about $20 \%$. Because there has been no report of the efficacy of weekly paclitaxel in the third-line setting, this retrospective study investigated the efficacy and toxicities of weekly paclitaxel used in the third-line setting for the treatment of gastric cancer refractory to all three key drugs, fluorouracil, irinotecan, and cisplatin, used in clinical practice.

Methods. In 85 patients with advanced or recurrent histologically confirmed gastric adenocarcinoma who had failed to respond to prior chemotherapy regimens containing fluorouracil, irinotecan, and cisplatin, paclitaxel $\left(80 \mathrm{mg} / \mathrm{m}^{2}\right)$ was administered weekly, three times, for 3 weeks out of 4 .

Results. The median number of courses was 3 (range, 1-38). The overall response rate was $23.2 \%(19 / 82)$ in the patients with measurable lesions, and ascites disappeared in 15 of 48 patients $(31.3 \%)$. Progression-free survival was 105 days and the median survival time was 201 days from the initiation of paclitaxel administration. Grade 3 or 4 leukopenia, neutropenia, anemia, and thrombocytopenia were observed in 25 $(29 \%), 25(29 \%), 37(44 \%)$, and $3(4 \%)$ patients. Other, nonhematological, toxicities were nausea, vomiting, anorexia, sensory neuropathy, fatigue, and febrile neutropenia.

Conclusion. Weekly paclitaxel administration shows activity against advanced gastric cancer also in the third-line setting.
\end{abstract}

Key words Metastatic gastric cancer - Weekly paclitaxel . Third-line chemotherapy

Offprint requests to: $\mathrm{H}$. Yasui

Received: July 9, 2009 / Accepted: September 2, 2009

\section{Introduction}

Gastric cancer remains the second leading cause of cancer death worldwide, with more than 700000 deaths per year [1]. In Japan, although markedly improved survival has been achieved, its mortality each year remains at approximately 50000, resulting in gastric cancer being the second-leading cause of cancer death in 2007 [2].

For patients with unresectable or recurrent gastric cancer, the main therapeutic option is palliative chemotherapy. Clinical trials of systemic chemotherapy for gastric cancer have shown significantly prolonged survival with the chemotherapy as compared to best supportive care [3-5]. Recently, two phase III studies have been reported from Japan. One was the Japan Clinical Oncology Group (JCOG) 9912 trial, which revealed the noninferiority of S-1 (a combined preparation of tegafur, 5-chloro-2,4-dihydroxypyridine, and potassium oxonate) alone to 5-fluorouracil (FU) alone and failed to demonstrate the superiority of irinotecan plus cisplatin (CDDP) to 5-FU alone in terms of the overall survival [6]. This trial concluded that 5 -FU could be replaced by S-1 in the chemotherapy for advanced gastric cancer. The other study was the S-1 plus cisplatin versus S-1 in RCT in the treatment for stomach cancer (SPIRITS) trial, which showed the superiority of S-1 plus CDDP to S-1 alone in terms of the overall survival [7]. Based on these trials, S-1 plus CDDP has been recognized as the standard first-line therapy for unresectable and recurrent gastric cancer in Japan.

No standard chemotherapy regimen has been established for use after the failure of first-line chemotherapy. For patients with failure in S-1+CDDP therapy, irinotecan and paclitaxel are considered as the key drugs for the subsequent chemotherapy. In clinical practice, two treatment strategies have generally been adopted after the failure of first-line chemotherapy; irinotecan or irinotecan combination chemotherapy as the 
second-line treatment followed by paclitaxel or paclitaxel combination chemotherapy as the third-line treatment, or vice versa.

A phase II study of paclitaxel for chemotherapynaive patients with gastric cancer showed a response rate (RR) of $23 \%$ and median survival time (MST) of 340 days, and this result led to the approval of paclitaxel for treating gastric cancer in Japan [8-11]. Paclitaxel may be administered by two methods, in a triweekly schedule and in a weekly schedule (3 weeks on and 1 week off). Studies of paclitaxel for ovarian, breast, and lung cancer treatment showed milder toxicities and equivalent activity of the drug when administered in the weekly schedule as compared with the triweekly schedule [12-16]. In Japan, weekly administration of paclitaxel for gastric cancer after the failure of first-line chemotherapy is very common, and a few trials have reported on the efficacy of weekly paclitaxel in patients with advanced or recurrent gastric cancer, especially in the second-line setting [17-23]. However, no clinical trials have investigated the effect of weekly paclitaxel in the third-line setting.

At our hospital, irinotecan was preferred for the second-line setting (unless the patient had a contraindication for the use of irinotecan (such as intestinal obstruction due to peritoneal dissemination) and subsequently weekly paclitaxel was selected for the third-line setting. In the present retrospective study, we investigated the efficacy and safety of weekly paclitaxel as third-line chemotherapy in patients with gastric cancer who were refractory to all the three key drugs, fluorouracil, CDDP, and irinotecan used in earlier settings.

\section{Patients and methods}

\section{Subjects}

In total, 119 patients with advanced or recurrent gastric cancer were treated with weekly paclitaxel in the thirdline setting between September 2002 and September 2008 at the Shizuoka Cancer Center, Shizuoka, Japan. Among them, the subjects of this retrospective study were 85 patients who were selected according to the following criteria: (1) histologically confirmed adenocarcinoma of the stomach; (2) failure of prior chemotherapy with at least two regimens, including 5-FU or its derivatives (S-1, capecitabine, tegafur/uracil [UFT]), irinotecan, and CDDP; (3) no history of prior chemotherapy with paclitaxel or docetaxel; (4) age 75 years or less; (5) performance status of 2 or less on the Eastern Cooperative Oncology Group scale; (6) adequate bone marrow, hepatic, and renal functions; (7) no synchronous double cancer or other serious disease; and (8) availability of informed consent before the start of treatment. The reasons that the remaining 34 patients were excluded from this study were: age in 3 patients, performance status in 3 , double cancer in 3 , nonadenocarcinoma in 3 , prior history of docetaxel in 2, history of local chemotherapy (CDDP intraperitoneal therapy) in 6 , and unknown details in 14 .

\section{Treatment}

Paclitaxel at $80 \mathrm{mg} / \mathrm{m}^{2}$ in $250 \mathrm{ml}$ normal saline was administered by intravenous infusion over $1 \mathrm{~h}$, and this was repeated weekly for 3 weeks out of 4 , on an outpatient basis as a rule. Short-term premedication was used to prevent paclitaxel-associated hypersensitivity reactions; dexamethasone $8 \mathrm{mg}$, diphenhydramine $50 \mathrm{mg}$, ranitidine $50 \mathrm{mg}$, and granisetron $3 \mathrm{mg}$ were administered $30 \mathrm{~min}$ before the infusion of paclitaxel. Treatment was repeated until disease progression, the occurrence of unacceptable toxicities, or the patient's refusal. In the event of serious hematological toxicity, treatment was suspended until recovery.

Although weekly administration of paclitaxel has not been approved in Japan, this schedule is widely used in clinical practice as a community standard. The clinical practice review committee in this hospital reviewed and approved this regimen for gastric cancer, and informed consent to receive this treatment was obtained from each patient.

\section{Response and toxicity evaluation}

Response was assessed every 2 months by computed tomography (CT). Objective responses in measurable metastatic lesions were evaluated according to the Response Evaluation Criteria in Solid Tumors (RECIST 1.0) [25]. Survival time was calculated from the date of initiation of paclitaxel to the date of death or last confirmation of survival. The efficacy for treating ascites was evaluated by the criteria of the Japanese classification of gastric carcinoma (13th edition). Symptomatic toxicity and laboratory data were monitored every week at the outpatient clinic. Toxicity was evaluated according to the Common Toxicity Criteria for Adverse Events, version 3.0 (CTCAE 3.0) [25].

\section{Results}

\section{Patients' backgrounds}

The patients' characteristics are shown in Table 1. Of the 85 patients, $63(74 \%)$ were male. The median age was 61 years (range, 21-75 years). Sixty-nine patients $(81 \%)$ showed a performance status of 0 or 1 . Forty-one patients (48\%) had primary lesions, 13 had pleural effu- 
Table 1. Patient characteristics $(n=85)$

\begin{tabular}{|c|c|c|}
\hline & Number & $\%$ \\
\hline \multicolumn{3}{|l|}{ Age (years) } \\
\hline Median (range) & $61(21-75)$ & \\
\hline \multicolumn{3}{|l|}{ Sex } \\
\hline Male & 63 & 74 \\
\hline Female & 22 & 26 \\
\hline \multicolumn{3}{|l|}{ Performance status (ECOG) } \\
\hline 0 & 26 & 31 \\
\hline 1 & 43 & 51 \\
\hline 2 & 16 & 18 \\
\hline \multicolumn{3}{|l|}{ Histology } \\
\hline Differentiated & 35 & 41 \\
\hline Undifferentiated & 47 & 55 \\
\hline Unknown & 3 & 4 \\
\hline \multicolumn{3}{|l|}{ Primary lesion } \\
\hline$(+)$ & 41 & 48 \\
\hline$(-)$ & 44 & 52 \\
\hline \multicolumn{3}{|l|}{ Metastatic sites } \\
\hline Lymph node & 49 & 58 \\
\hline Peritoneum & 41 & 48 \\
\hline Liver & 34 & 40 \\
\hline Ovary & 12 & 14 \\
\hline Lung & 11 & 13 \\
\hline Bone & 5 & 6 \\
\hline \multicolumn{3}{|l|}{ Number of metastatic sites } \\
\hline One & 35 & 41 \\
\hline Two & 29 & 34 \\
\hline Three or more & 21 & 25 \\
\hline Pleural effusion & 13 & 15 \\
\hline Ascites & 48 & 56 \\
\hline \multicolumn{3}{|l|}{ Prior chemotherapy } \\
\hline \multicolumn{3}{|l|}{ First-line regimen $(n=85)$} \\
\hline Oral fluoropyrimidine & 57 & 67 \\
\hline 5-FU (i.v.) & 14 & 16 \\
\hline CPT-11 & 14 & 16 \\
\hline \multicolumn{3}{|l|}{ Second-line regimen $(n=85)$} \\
\hline Oral fluoropyrimidine & 16 & 19 \\
\hline 5-FU (i.v.) & 3 & 4 \\
\hline СРТ-11 & 66 & 77 \\
\hline \multicolumn{3}{|l|}{ Third-line regimen $(n=5)$} \\
\hline CPT-11 & 4 & 80 \\
\hline Other & 1 & 20 \\
\hline \multicolumn{3}{|l|}{ Number of prior regimens } \\
\hline One & 80 & 94 \\
\hline Three & 5 & 6 \\
\hline \multicolumn{3}{|c|}{ Subsequent chemotherapy after paclitaxel } \\
\hline None (best supportive care) & 38 & 45 \\
\hline One regimen & 36 & 42 \\
\hline Two regimens & 7 & 8 \\
\hline Three regimens or more & 4 & 5 \\
\hline
\end{tabular}

ECOG, Eastern Cooperative Oncology Group; 5-FU, 5-fluorouracil; CPT-11, irinotecan

sion, and 48 had ascites. The number of sites affected by metastasis, including lymph node, peritoneum, liver, ovary, lung and bone, was one in 35 patients, two in 29 patients, and three or more in 21 patients. All patients had received prior chemotherapies with regimens containing fluorouracil or its derivatives, irinotecan, and CDDP.
Table 2. Toxicity $(n=85)$

\begin{tabular}{lrrrrr}
\hline & \multicolumn{5}{c}{ Grade } \\
\cline { 2 - 6 } & 1 & 2 & 3 & 4 & $3 / 4(\%)$ \\
\hline Hematological & & & & & \\
$\quad$ Leukopenia & 21 & 17 & 23 & 2 & 29 \\
Neutropenia & 4 & 14 & 19 & 6 & 29 \\
Anemia & 13 & 36 & 23 & 12 & 41 \\
Thrombocytopenia & 9 & 6 & 1 & 2 & 4 \\
Nonhematological & & & & & \\
Nausea & 10 & 2 & 2 & 0 & 2 \\
Vomiting & 5 & 7 & 2 & 0 & 2 \\
Diarrhea & 12 & 2 & 0 & 0 & 0 \\
Anorexia & 16 & 11 & 2 & 0 & 2 \\
Mucositis & 6 & 0 & 0 & 0 & 0 \\
Sensory neuropathy & 33 & 9 & 1 & 0 & 1 \\
Motor neuropathy & 1 & 3 & 2 & 0 & 2 \\
Edema & 7 & 0 & 0 & 0 & 0 \\
Allergic reaction & 1 & 0 & 0 & 0 & 0 \\
Fatigue & 19 & 10 & 2 & 0 & 2 \\
Febrile neutropenia & - & - & 7 & 0 & 8 \\
\hline
\end{tabular}

\section{Dose intensity}

The total number of paclitaxel infusions was 870 . The median number of courses per patient was 3 (range, 138). The dose intensity was calculated as $51.4 \mathrm{mg} / \mathrm{m}^{2}$ per week, which corresponded to $86 \%$ of the planned dose. The dose was reduced in 9 patients; because of myelosuppression in 6, hepatic and renal dysfunction in 1, mucositis in 1 , and poor general condition in 1 . The treatment was discontinued in all patients: due to disease progression in 79 patients, development of neutropenia in 4 , development of neuropathy in 1 , and comorbidity (cerebral infarction) in 1 patient.

\section{Toxicity}

The hematological and nonhematological toxicities encountered are shown in Table 2. Hematological toxicities were common, and 25 (29\%) patients experienced grade 3 or 4 leukopenia and neutropenia. Seven (8\%) patients developed febrile neutropenia. Anemia was the most common adverse event, because 82 of the $85(96 \%)$ patients had had anemia before the initiation of paclitaxel, including 18 patients with grade 3 or 4 anemia. Thirty five patients (41\%) experienced grade 3 or 4 anemia, with an incidence of grade 3 or 4 thrombocytopenia of only $4 \%$. As nonhematological toxicities, 2 patients ( $2 \%$ ) experienced grade 3 nausea/vomiting and anorexia. Grade 3 sensory neuropathy was observed in 1 patient $(1 \%)$ and motor neuropathy occurred in 2 patients $(2 \%)$. No severe allergic reactions were reported. One patient (1\%) died within 30 days of the last administration of paclitaxel after disease progression was confirmed. 


\section{Overall survival}

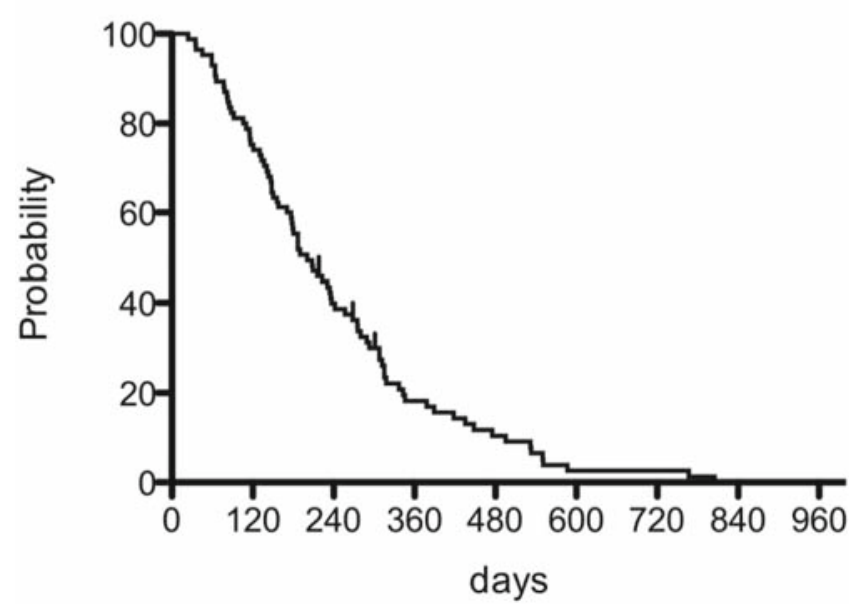

Fig. 1. Overall survival

Table 3. Response to weekly paclitaxel $(n=82)$

\begin{tabular}{lcc}
\hline Response & Number of patients & $\%$ \\
\hline CR & 0 & \\
PR & 19 & 23.2 \\
SD & 35 & 42.7 \\
PD & 27 & 32.9 \\
NE & 1 &
\end{tabular}

CR, complete response; PR, partial response; $\mathrm{SD}$, stable disease; $\mathrm{PD}$, progressive disease; NE, not evaluable

\section{Responses and survival}

Eighty-two of the 85 patients were assessable for response, and the remaining 3 patients did not have measurable disease. Nineteen of the 82 patients $(23 \%)$ showed a partial response, yielding a response rate of $23.2 \%$ (Table 3) and a disease control rate (partial response + stable disease) of $65.9 \%$. Ascites disappeared in 7 patients and decreased in size in $8(31.3 \%)$ of the 48 patients who had been noted to have ascites before the start of treatment. The median follow-up period was 561 days when the survival data were updated in March 2009. The median survival time was 201 days after the initiation of paclitaxel administration (Fig. 1). The median progression-free survival (PFS) was 105 days (Fig. 2). After the failure of paclitaxel, 38 patients $(45 \%)$ received no further chemotherapy or showed disease progression, 36 received one regimen of subsequent chemotherapy, 7 received two regimens, and 4 received three or more regimens.

\section{Discussion}

This retrospective study investigated the efficacy and tolerability of weekly paclitaxel in patients with heavily

\section{Progression free survival}

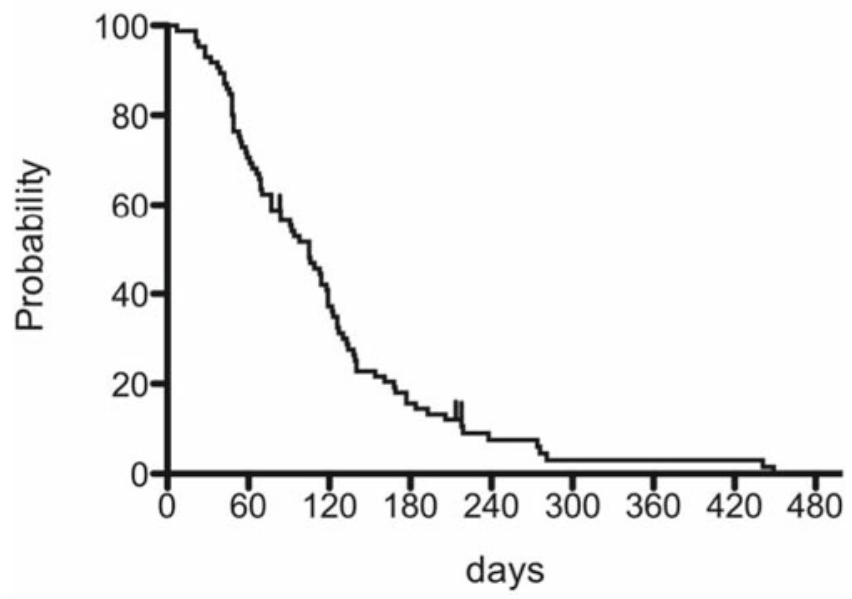

Fig. 2. Progression-free survival

treated advanced gastric cancer. Weekly paclitaxel is one of the most commonly used regimens in the secondline or later setting for gastric cancer in Japan. Five reports of weekly paclitaxel for gastric cancer have revealed similar response rates, of about $15.9 \%-33.3 \%$, and similar median survival times, of 5.0-7.8 months, despite the differences in the patient background characteristics in each of the studies. The present study, in which all the patients had received two or more treatment regimens containing 5-FU, irinotecan, and cisplatin, showed a response rate of $23 \%$ and median survival time of 6.7 months. These results seem to be similar to those of previous studies in the first- or second-line setting. Thus, it is speculated that paclitaxel may show consistent efficacy, irrespective of the previous chemotherapy.

Peritoneal dissemination is a major and serious complication of advanced gastric cancer, often resulting in the development of ascites, intestinal obstruction, and hydronephrosis, especially after failure of chemotherapy. Most patients with peritoneal dissemination or malignant ascites are usually excluded from clinical trials because of the lack of a measureable lesion or their poor general medical condition; hence, the efficacy of chemotherapy for peritoneal dissemination has not yet been confirmed. It has been reported that paclitaxel reaches an effective concentration for the treatment of ascites $(8.5 \mathrm{ng} / \mathrm{ml})$ when administered by a weekly schedule [26-29]. In line with several reports of the efficacy of paclitaxel for malignant ascites, showing consistent efficacy [26-28], the present study showed a proportion of patients with decrease or remission of ascites $(31.3 \%)$ similar to that shown in previous studies $[19,21]$. These results may lend support to the notion that paclitaxel still remains effective against malignant ascites in the third-line setting, while irinotecan cannot 
be used for patients with severe peritoneal dissemination for fear of severe toxicities.

The greatest concern in third-line chemotherapy for cancer is drug toxicity. The incidences of grade 3 or 4 leukopenia, neutropenia, and anemia in the present study were $29 \%, 29 \%$, and $44 \%$, with $8 \%$ developing febrile neutropenia. These percentages appear to be rather high as compared to those reported previously [19], while the incidence of severe nonhematological toxicities of $2 \%$ or less appeared to be consistent with that in previous studies. Especially, anemia was the most frequently encountered severe toxicity in the present study. All of the subjects in this study had been heavily treated before, and chronic anemia due to bleeding from the primary lesion may have occurred in some of the 41 patients (48\%) with primary lesions. Indeed, 82 of the $85(96 \%)$ patients were found to have grade 1 or more severe anemia and $18(22 \%)$ had grade 3 or 4 anemia immediately before the start of treatment. Yamada et al. [11] reported an incidence of grade1 or 2 peripheral neuropathy of $73 \%$ (44/60) with triweekly paclitaxel therapy. In our present study, the incidence of grade 3 peripheral neuropathy was as low as $4 \%$, and that of grade 1 or 2 was $54 \%$. Short duration of treatment and administration by a weekly schedule may explain the low incidence of neurotoxicity in this study. It is considered that the use of weekly paclitaxel in the third-line setting may be feasible, but it requires careful management of any hematological toxicities.

In conclusion, weekly paclitaxel administration seems to be feasible and show activity for advanced gastric cancer also in the third-line setting. Although careful management of hematological toxicities is required, this therapy can be applied even for patients with severe peritoneal dissemination. Weekly paclitaxel therapy in the third-line setting may be one of the feasible therapeutic strategies for metastatic and recurrent gastric cancer.

\section{References}

1. Parki DM, Bray F, Ferlay J, Pisani P. Global cancer statistics, 2002. CA Cancer J Clin 2005;55:74-108.

2. Statistics and Information Department, Ministry of Health, Labour and Welfare. Vital statistics in 2007 (in Japanese). http://www. mhlw.go.jp/. Accessed 30 April 2009.

3. Pyrhoenen S, Kuitunen T, Nyandoto P, Kouri M. Randomized comparison of fluorouracil, epidoxorubicin and methotrexate (FEMTX) plus supportive care with supportive care alone in patients with non-resectable gastric cancer. Br J Cancer 1995;71: 587-91.

4. Murad AM, Santiago FF, Petroianu A, Rocha PR, Rodrigues MA, Rausch M. Modified therapy with 5-fluorouracil, doxorubicin, and methotrexate in advanced gastric cancer. Cancer 1993;72:37-41.

5. Glimelius B, Hoffman K, Haglund U, Nyren O, Sjoeden PO Initial or delayed chemotherapy with best supportive care in advanced gastric cancer. Ann Oncol 1994;5:189-90.
6. Boku N, Yamamoto S, Shirao K, Doi T, Sawaki A, Koizumi W, et al. Gastrointestinal Oncology Study Group/Japan Clinical Oncology Group. Randomized phase III study of 5-fluorouracil (5-FU) alone versus combination of irinotecan and cisplatin (CP) versus S-1 alone in advanced gastric cancer (JCOG 9912). J Clin Oncol 2007;25(18S):LBA4513.

7. Koizumi W, Narahara H, Hara $T$, Takagane $T$, Takagi $M$, Miyashita K, et al. S-1 plus cisplatin versus S-1 alone for first-line treatment of advanced gastric cancer (SPIRITS trial): a phase III trial. Lancet Oncol 2008;9:215-21.

8. Ajani JA, Fairweather J, Dumas P, Patt YZ, Pazdur R, Mansfield PF. Phase II study of Taxol in patients with advanced gastric carcinoma. Cancer J Sci Am 1998;4:269-74.

9. Cascinu S, Graziano F, Cardarelli N, Marcellini M, Giordano P, Menichetti ET, et al. Phase II study of paclitaxel in pretreated advanced gastric cancer. Anticancer Drugs 1998;9:307-10.

10. Ohtsu A, Boku N Tamura F, Muro K, Shimada Y, Saigenji K, et al. An early phase II study of a 3-hour infusion of paclitaxel for advanced gastric cancer. Am J Clin Oncol 1998;21:416-9.

11. Yamada Y, Shirao K, Ohtsu A, Boku N, Hyodo I, Saitoh H, et al. Phase II trial of paclitaxel by 3-hour infusion for advanced gastric cancer with short premedication for prophylaxis against paclitaxel-associated hypersensitivity reactions. Ann Oncol 2001;12:1133-7.

12. Glantz MJ, Choy H, Akerley W, Kearns CM, Egorin MJ, Rhodes $\mathrm{CH}$, et al. Weekly paclitaxel with and without concurrent radiation therapy: toxicity, pharmacokinetics, and response: Semin Oncol 1996;23(6 Suppl 16):128-35.

13. Fennelly D, Aghajanian C, Shapiro F, O'Flaherty C, McKenzie $\mathrm{M}$, O'Connor C, et al. Phase I and pharmacologic study of paclitaxel administered weekly in patients with relapsed ovarian cancer. J Clin Oncol 1997;15:187-92.

14. Rosenberg P, Anderson H, Boman K, Ridderheim M, Sorbe B, Puistola U, et al: Randomized trial of single agent paclitaxel given weekly versus every 3 weeks and with peroral versus intravenous steroid premedication to patients with ovarian cancer previously treated with platinum. Acta Oncol 2002;41:418-24.

15. Seidman AD, Berry D, Cirrincione C, Harris L, Muss H, Marcom PK, et al. Randomized phase III trial of weekly compared with every-3-weeks paclitaxel for metastatic breast cancer, with trastuzumab for all HER-2 overexpressors and random assignment to trastuzumab or not in HER-2 nonoverexpressors: final results of Cancer and Leukemia Group B Protocol 9840. J Clin Oncol 2008;26:1642-49.

16. Akerley W, Glantz M, Choy H, Rege V, Sambandam S, Joseph $\mathrm{P}$, et al. Phase I trial of weekly paclitaxel in advanced lung cancer. J Clin Oncol 1998;16:153-8.

17. Cho H, Shotsu A, Konishi K, Kanari M, Tsuburaya A, Kobayasi $\mathrm{O}$, et al. Weekly paclitaxel for the second-line treatment of recurrent and advanced gastric cancer (RAGC). Proc Am Soc Clin Oncol 2002;21 (abstract 2365).

18. Arai T, Hamaguchi T, Shirao K, Yamada Y, Muro K, Matsumura $\mathrm{Y}$, et al. Weekly paclitaxel in patients with heavily treated advanced gastric cancer. Proc Am Soc Clin Oncol 2003;22 (abstract 1291).

19. Arai T, Hamaguchi T, Shirao K, Shimada Y, Yamada Y, Muro $\mathrm{K}$, et al. Weekly paclitaxel (PTX) in patients with heavily treated advanced gastric cancer (AGC). Jpn J Cancer Clin 2003;49: $621-5$.

20. Emi Y, Kakeji Y, Baba H, Ishida T, Maehara Y. Weekly paclitaxel therapy as second-line therapy for advanced, recurrent stomach cancer. Jpn J Cancer Clin 2004;50:277-81.

21. Hironaka S, Zenda S, Boku N, Fukutomi A, Yoshimo T, Onozawa Y. Weekly paclitaxel as second-line chemotherapy for advanced or recurrent gastric cancer. Gastric Cancer 2006;9:14-8.

22. Kodera Y, Ito S, Mochizuki Y, Fujitake S, Koshikawa K, Kanyama $\mathrm{Y}$, et al. A phase II study of weekly paclitaxel as second-line chemotherapy for advanced gastric cancer (CCOG 0302 study). Anticancer Res 2007;27:2667-72. 
23. Emi Y, Yamamoto M, Takahashi I, Orita H, Kakeji Y, Kohnoe S, et al. Phase II study of weekly paclitaxel by 1-hour infusion for advanced gastric cancer. Surg Today 2008;38:1013-20.

24. Japanese translation of common terminology criteria for adverse events (CTCAE), and instructions and guidelines. Int $\mathrm{J}$ Clin Oncol 2004;9(Suppl 3):1-82.

25. Gehan EA, Tefft MC. Will there be resistance to the RECIST (response evaluation criteria in solid tumors)? J Natl Cancer Inst 2000;92:179-81.

26. Kobayashi M, Sakamoto J, Namikawa T, Okamoto K, Okabayashi $\mathrm{T}$, Ichikawa $\mathrm{K}$, et al. Pharmacokinetic study of paclitaxel in malignant ascites from advanced gastric cancer patients. World $\mathrm{J}$ Gastroenterol 2006;12:1412-5
27. Ishida T, Shimokawa H, Kawaguchi K, Nose N, Ikegami T, Itoh $\mathrm{H}$, et al. Effective weekly paclitaxel administration for gastric cancer with malignant ascites: a case report (in Japanese). Jpn J Cancer Chemother (Gan To Kagaku Ryoho) 2002;29:1643-46.

28. Hikino H, Yamada T, Hata T, Nakamura $K$, Sugimoto $S$, Kanazawa A, et al. Efficacy of weekly administration of paclitaxel for advanced or recurrent gastric cancer with peritoneal dissemination. Jpn J Cancer Chemother 2004;31:759-61.

29. Kojima T, Matsui T, Uemura T, Fujimitsu Y, Kato J, Kojima H. A case of advanced gastric cancer treated with weekly paclitaxel with measurement of concentrations in blood and ascites. Jpn J Cancer Chemother 2003;30:1529-31. 